\title{
Solar activity explored with new wavelet methods
}

\author{
H. Lundstedt ${ }^{1}$, L. Liszka ${ }^{2}$, and R. Lundin ${ }^{3}$ \\ ${ }^{1}$ Swedish Institute of Space Physics, Lund, Sweden \\ ${ }^{2}$ Swedish Institute of Space Physics, Umeå, Sweden \\ ${ }^{3}$ Swedish Institute of Space Physics, Kiruna, Sweden
}

Received: 1 June 2004 - Revised: 8 March 2005 - Accepted: 22 March 2005 - Published: 3 June 2005

\begin{abstract}
In order to improve the forecasts of the impact of solar activity on the terrestrial environment on time scales longer than days, improved understanding and forecasts of the solar activity are needed. The first results of a new approach of modelling and forecasting solar activity are presented. Time series of solar activity indicators, such as sunspot number, group sunspot number, F10.7, E10.7, solar magnetic mean field, Mount Wilson plage and sunspot index, have been studied with new wavelet methods; ampligrams and time-scale spectra. Wavelet power spectra of the sunspot number for the period 1610 up to the present show not only that a dramatic increase in the solar activity took place after 1940 but also that an interesting change occurred in 1990. The main 11-year solar cycle was further studied with ampligrams for the period after 1850 . time-scale spectra were used to examine the processes behind the variability of the solar activity. Several interesting deterministic and more stochastic features were detected in the time series of the solar activity indicators. The solar nature of these features will be further studied.
\end{abstract}

Keywords. Solar physics, astrophysics and astronomy (Magnetic fields; Stellar interiors and dynamo theory) Space plasma physics (nonlinear phenomena)

\section{Introduction}

Space weather, which is driven by solar activity, can have a severe effect on technological systems (Lundstedt, 2003). Recent research shows a closer relation exists between solar activity and climate changes than previously thought. Boberg and Lundstedt (2002) showed the variation of the North Atlantic Oscillation (NAO) index to be correlated with the electric field strength of the solar wind for the period 1973 to 2000. Shindell et al., (2001) showed that the cold climate during the Maunder minimum could be related to a lowered solar induced UV radiation, ozone and a NAO value. The earth's cloud coverage has also been shown to be affected by

Correspondence to: $\mathrm{H}$. Lundstedt

(henrik@lund.irf.se) cosmic ray flux and thus correlated to solar activity (Svensmark and Friis-Christensen, 1997).

Since the solar activity can be described as a nonlinear chaotic dynamic system (Mundt et al., 1991) methods such as neural networks and wavelet methods should be very suitable (Lundstedt (1997); Lundstedt (2001)). Many have used wavelet techniques for studying solar activity. Frick et al. (1997) investigated the wavelet transform of the monthly sunspot group number from 1610 to 1994 . Two pronounced peaks were found corresponding to the Schwabe (11 years) and Gleissberg (90-100 years) cycles. The wavelet analysis also showed the Maunder and Dalton minima. Knaack and Stenflo (2002) first calculated the spherical coefficients of the radial magnetic field from Kitt Peak Observatory maps. They then applied wavelet analysis to deduce the temporal variation. The wavelet power spectra of the spherical coefficients showed power for 22 years, 6-7 years, and for 2-3 years.

In Boberg et al., (2002) we used both the daily solar mean magnetic field (SMMF) observed at the Wilcox Solar Observatory (Stanford) and one-minute resolution measurements of SMMF by MDI on board SOHO. Peaks were found in the power for periods of 11 years (the solar cycle), 1-2 years (related to variations in internal rotation), 80-200 days (related to evolution of active regions) and 13/26 days (related to solar rotation). Using one-minute resolution SMMF we managed to detect peaks in power around $90 \mathrm{~min}$. Further studies suggested that the 90-min oscillation could be associated with the occurrence of CMEs. Polygiannakis et al. (2003) used the sunspot number $R_{z}$ and Krivova and Solanki (2002) used the sunspot area (SA) as an indicator of the solar activity in a similar wavelet study. Polygiannakis et al. (2003), tried to explain the periodicities found as a result of two dynamos with periods of 11 and 2 years. Krinova and Solanki, on the other hand, suggest that the 1.3-year and 156-day periods are harmonics of the solar activity cycle. Richardsson et al. (1994) found 1.3-year periodicities in the solar wind variation.

Oliver and Ballester (2002) found 158-day periodicities in the number of X-ray flares, sunspot number, sunspot area and the MWSI. However, they noticed that the amplitude varies from cycle to cycle. 


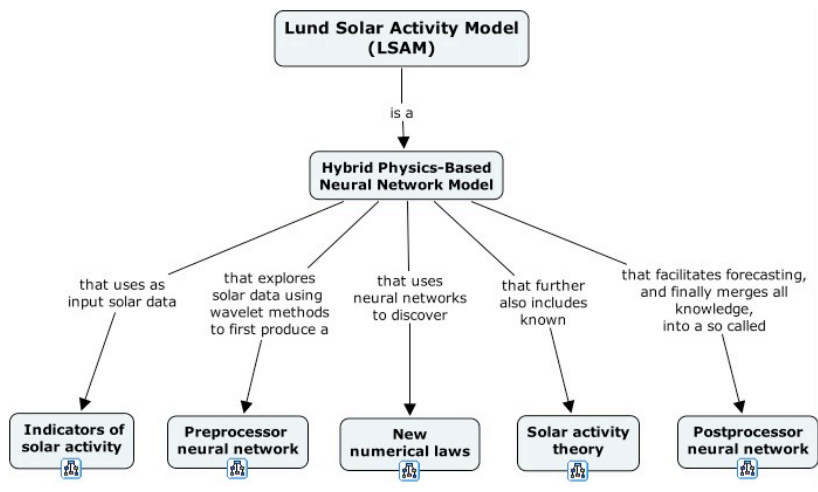

Fig. 1. The scientific approach of our program.

In this article we outline a new approach of exploring, explaining and forecasting solar activity, based on new wavelet methods and physics-based neural networks using solar results on the solar dynamo and from helioseismology (Fig. 1) (Lundstedt, 2004). We begin by describing the indicators of solar activity (Rabin et al., 1991) that are used in this study. New wavelet methods are introduced, so-called ampligrams and time-scale spectra (Wernik et al., (1997); Liszka (2003)). These new methods are then applied to the solar activity indicators. Scalograms, ampligrams and time-scale spectra show not only the periodicities, and amplitudes but also the processes behind the variability. We then try to interpret these features using solar theory and cognitive methods. Finally, forecasts and future work are described.

\section{Data - indicators of solar activity}

In this study we have used daily values of the following indicators of solar activity: The sunspot number $R_{z}\left(R_{z}=k(10 g+f)\right)$, where $\mathrm{g}$ is the number of sunspot groups, $f$ the number of individual sunspots, and $\mathrm{k}$ a correction factor depending on the observer. The sunspot group number $R_{g}, R_{g}=\left(\frac{12.08}{n} \sum k G\right)$ (Hoyt and Schatten, 1998), where $\mathrm{n}$ is the number of observers, $\mathrm{G}$ the number of sunspot groups and $\mathrm{k}$ a correction factor. It is a manifestation of an east-west magnet produced by the stretching of an initial poloidal north-south field under the effect of a non uniform rotation. The sunspots are confined to belts, which extend to about 35 degrees latitude on either side of the solar equator. The F10.7 radio intensity (Rabin et al., 1991) consists of emission from three sources, including the undisturbed solar surface, developing active regions and short-lived enhancements above the daily level. The E10.7 (Tobiska, 2001) is a proxy for the daily extreme ultraviolet (EUV) irradiance. The solar mean magnetic field (SMMF), Boberg et al. (2002); Scherrer et al. (1977), is defined as the average field over the entire visible solar disk. The SMMF is a measure of the magnetic field strength outside active regions, since active regions have, to a large extent, equal amounts of positive and negative magnetic fluxes. For each magnetogram taken at the 150-foot solar tower, a Magnetic Plage Strength In- dex (MPSI) value and a Mt. Wilson Sunspot Index (MWSI) value are calculated. To determine MPSI they sum the absolute values of the magnetic field strengths for all pixels where the absolute value of the magnetic field strength is between 10 and 100 gauss. This number is then divided by the total number of pixels (regardless of magnetic field strength) in the magnetogram. The MWSI values are determined in much the same manner as the MPSI, though summation is only done for pixels where the absolute value of the magnetic field strength is greater than 100 gauss. The missing data $(2 \%)$ have been reconstructed and time series converted to equidistant using the cubic splines interpolation.

\section{Wavelet methods}

Today using wavelet techniques has become a common method of analysing solar-terrestrial data. Good introductions to the use of wavelet transforms are given by Torrence and Compo (1998) and Kumar and Foufoula-Georgiou (1997). Wavelet analysis is a powerful tool both to find the dominant mode of variation and also to study how it varies with time, by decomposing a nonlinear time series into timefrequency space. The wavelet transform of a function $y(t)$ is given by

$w(a, b)=a^{-1 / 2} \int_{-\infty}^{+\infty} y(t) g^{*}\left(\frac{t-b}{a}\right) d t$,

where $\mathrm{a}$ is the scale dilation, i.e. the compressing and stretching of the wavelet $\mathrm{g}, \mathrm{b}$ is the translation parameter, i.e. the shifting of $\mathrm{g}$, and $g^{*}$ the complex conjugate of $\mathrm{g}$. The Morlet wavelet is defined as a complex sine wave, localized with a Gaussian and given by

$g(t)=\exp \left(i \omega_{0} t-\frac{t^{2}}{2}\right)$,

where $\omega_{0}$ is a phase constant. To analyze a discrete signal $y\left(t_{i}\right)$ we need to sample the continuous wavelet transform on a grid in the time-scale plane (b,a). By setting $a=j$ and $b=k$ the wavelet coefficients $w_{j, k}$ are

$w_{j, k}=j^{-1 / 2} \int_{-\infty}^{+\infty} y(t) g^{*}\left(\frac{t-k}{j}\right) d t$.

When the wavelet coefficient magnitudes (WCM) are plotted for the scale and the elapsed time, a so-called scalogram is produced. Many times series observed in physics consist of a deterministic part with a superimposed stochastic component. Wernik et al. (1997) introduced a method to study this, based on non-linear filtering of the wavelet coefficients. The deterministic strong part is obtained by setting to zero all wavelet coefficients less than a certain threshold. The inverse wavelet transform is then used to calculate the corresponding time series. The stochastic weak part is obtained by setting to zero all wavelet coefficients greater than that threshold level. The inverse wavelet transform is then used to calculate the 
corresponding time series. New wavelet spectra are finally calculated for each partial time series.

Ampligrams and time-scale spectra (Liszka, 2003) can be looked upon as a generalization of the above technique, a kind of band-pass filtering in the WCM domain analogous to Fourier analysis is in a frequency domain. They can be used to separate independent components of the signal, assuming that the different components are characterized by different wavelet coefficient magnitudes (spectral densities). Ampligrams are constructed in the following way: The maximum magnitude $(|W|)$ among the wavelet coefficients is first found. L magnitude intervals are then defined,

$I_{l}=[(l-1) \Delta w, l \Delta w], l=1,2 . . L$ with $\Delta \mathrm{w}=\frac{|\mathrm{W}|}{\mathrm{L}}$.

From that we construct $\mathrm{L}$ matrices $\mathbf{W}_{l}, \mathrm{l}=1,2 \ldots \mathrm{L}$ such that

$w_{j, k}^{(l)}= \begin{cases}w_{j, k} & \text { if }\left|w_{j, k}\right| \in I_{l} \\ 0 & \text { otherwise. }\end{cases}$

Inverse the wavelet transform to get a new time-signal $y_{l}\left(t_{i}\right), l=1, . . L$. Each $y_{l}\left(t_{i}\right)$ is what the signal should have looked like if only a narrow range of wavelet coefficient amplitude would be present in the signal. After that an L X N matrix $\mathbf{Y}$ is constructed with $y_{l}\left(t_{i}\right)$ as rows. This matrix $\mathbf{Y}$ is the ampligram of the original time-signal $y_{l}\left(t_{i}\right)$.

Finally to construct the time-scale spectra each row of the ampligram matrix $\mathbf{Y}$ is first wavelet transformed, which results in L matrices. We then time-average these matrices (average along rows) leading to $\mathrm{L}$ arrays $\bar{w}_{l}$ with $\mathrm{J}$ elements. Finally, an L X J matrix $\overline{\mathbf{Y}}$ is constructed, with $\bar{w}_{l}$ as rows. This matrix $\overline{\mathbf{Y}}$ is the time-scale spectrum of the ampligram.

\section{Results}

\subsection{Wavelet power spectra, WCM and ampligrams}

We started by applying the well-known wavelet tools, developed by Torrence and Compo (1998), on the yearly group sunspot number (1610-1995) and sunspot number (1995 up to 2004). Upper panel of Fig. 2 shows the yearly values. The Maunder minimum (1645-1715) and Dalton minimum (about 1800) are seen. The middle panel shows the wavelet power spectra, with a cone of avoidance and contours enclosing regions of greater than $95 \%$ confidence level. The main 11-year solar cycle for years 1850 up to 2004 is of $95 \%$ confidence. The lower panel shows the average variance. The Maunder minimum and the Dalton minimum are clearly seen as well as the dramatic change around 1940.

We then examined the WCM, see Eq. (3) in more detail for the period 1850 up to the present in a scalogram. The maximum wavelet coefficient magnitude of the main periodicity of the solar cycle was calculated. In Fig. 3 we show the relation between the WCM maximum and the simultaneous time-scale. The well-known relation between the solar activity cycle length and amplitude is clearly seen. Frick et al. (1997) showed interestingly that the length of the solar
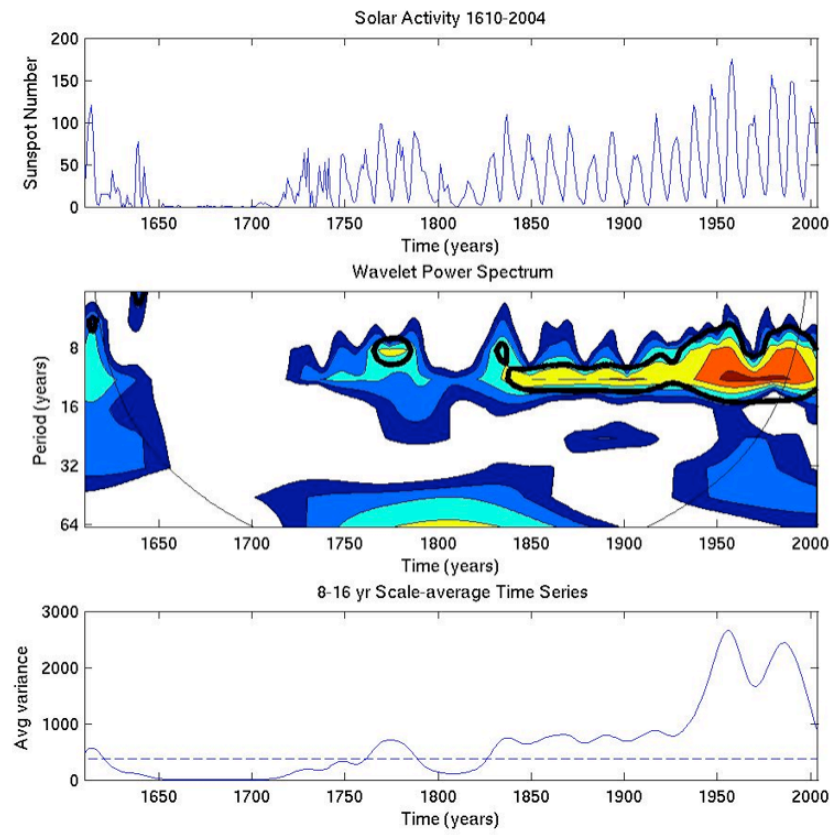

Fig. 2. Upper panel shows the group sunspot number from 1610 to 1995 and the sunspot number up to 2004. Middle panel shows the wavelet power spectra, with a cone of avoidance and enclosed regions of greater than $95 \%$ confidence level (thick black solid line). Lower panel shows the average variance. The Maunder and Dalton minima are clearly seen as well as the dramatic change after about 1940.

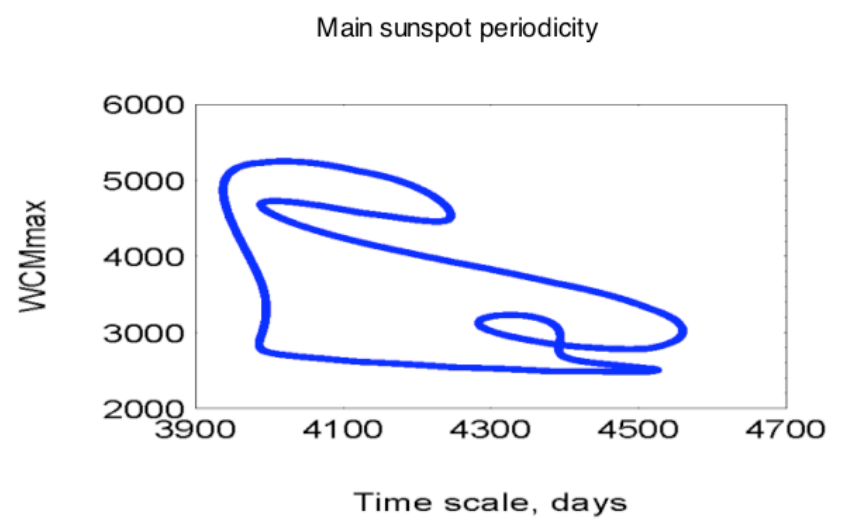

Fig. 3. The relation between the WCMmax and the simultaneous time scales for the main periodicity of the solar cycle.

cycle dramatically increased just before the Maunder minimum. However, Fig. 3. also shows a more complicated, chaotic picture. This will be further studied.

It has been claimed (Usoskin et al., 2003) that the Sun has never been as active as after 1940 during the last 1000 years. However we also notice a decrease in activity in 1990. In Fig. 2 the lower panel, the solar activity seems to dramatically drop around 1990. Is this a trend? The drop is, however, just at the limit of the cone of avoidance in the middle panel of Fig. 2. We therefore examined if any other observa- 


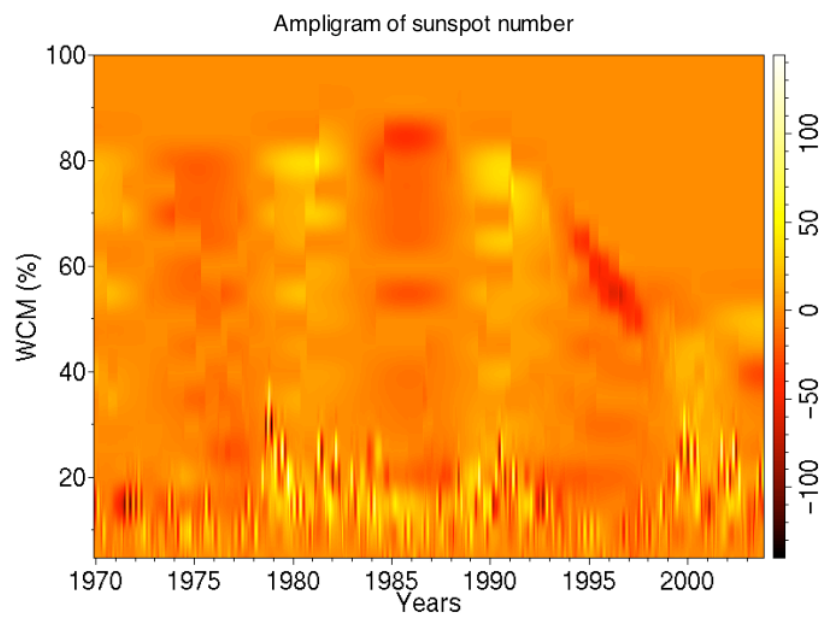

Fig. 4. The ampligram of the sunspot number from 1970 to 2004. WCM (\%) stands for the fraction of the maximum of the wavelet coefficient magnitude.

tions could support the trend. The same trend was also found for F10.7 and E10.7. Figure 3 shows an ampligram of the sunspot number from 1970 to 2004.

It's interesting to notice that the drop starts earlier for a higher fraction of the maximum of the wavelet coefficient magnitude. Scientists are now already predicting the amplitude of the next solar cycle 24, Duhau (2003); Kane (2002); Schatten (2002); Hathaway et al. (2003); Svalgaard et al. (2004). Most expect a rather small cycle 24, i.e. in accordance with the trend. However, Hathaway et al., 2003 claim that cycle 24 will become strong because the meridional circulation for cycle 22 was fast.

\subsection{Time-scale spectra}

The interesting property of the time-scale spectra is that deterministic periodic or semi-periodic structures in the data are mapped on the graph as vertically elongated features, while purely stochastic structures are mapped as horizontally elongated features. That property of the time-scale spectrum may be illustrated as follows: A pure and stationary sine-like signal will be mapped on the time-scale spectrum as a single dot. Introducing random phase variations, but without changing the signal amplitude will broaden the dot in the horizontal direction. On the other hand, introducing random amplitude fluctuations, without scrambling the phase, will broaden the dot in the vertical direction.

In Fig. 5 (upper panel) we present time-scale spectra of the sunspot number $R_{z}$ for the period 1850 to 2002 and 1975 to 2000 in Fig. 5 (lower panel). During the whole period the 11 -year time-scale (about 4000 days) solar cycle period is dominating. Both the amplitude and the time-scale are extended. The amplitude (monthly mean $R_{z}$ ) of the solar maxima ranged from 64 to 201 for the cycles 10 to 23 (NOAA and SEC). The length of the solar cycles ranged from 9.7 to 12.1 years (NOAA and SEC). Interesting to notice is also

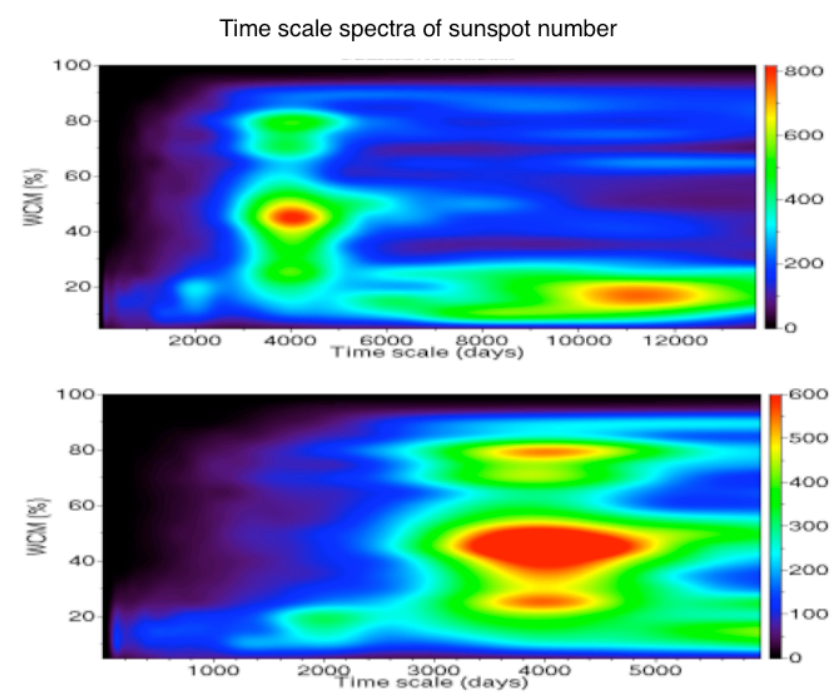

Fig. 5. time-scale spectra of sunspot number for the period 1850 to 2002 (upper panel) and 1975 to 2002 (lower panel).

the very extended horizontally weak (WCM $\approx 20 \%$ ) feature centered around 31-33 years. This 30-year signal has also been detected in works by, for example, Clua de Gonzalez et al. (1993). The solar nature of it is unclear. The time-scale spectrum of the sunspot number for 1975 to 2002 shows the main 11-year periodicity cycles as separate features due to their different amplitudes. A weaker feature $(\mathrm{WCM} \approx 20 \%)$ at about 2000 days is visible as well as for the whole period.

In Fig. 6 we also show time-scale spectra for the following solar activity indicators: F10.7, E10.7, MPSI and MWSI for the period 1975 to 2002 . The time-scale spectrum of F10.7 (upper left) shows again a strong 11 years feature and also weaker (WCM $\approx 20 \%$ ) features at about 3000 and 2000 days. The time-scale spectrum of E10.7 (upper right) shows very similar features as for F10.7. The different 11-year cycles are not so pronounced. The time-scale spectrum of the Mount Wilson sunspot solar magnetic field index (MWSI) (lower left) shows mostly a feature at 11 years. The timescale spectrum of the Mount Wilson sunspot field (MPSI) (lower right) shows, on the other hand, many features besides the 11-year feature. It's interesting to notice that again at least two 11-year cycles are seen as separate features, one centered at somewhat shorter time scales but stronger than the other. We also notice extended weaker features at WCM $\approx 40 \%$ and $\mathrm{WCM} \approx 20 \%$. The solar nature of all these interesting features will be studied further.

Solar magnetograms are clearly the best indicators of solar activity to explore. They show many features, which are more directly related to solar phenomena. In Boberg et al., (2002) we studied WSO and SOHO magnetograms in order to investigate solar activity on shorter terms, two years and less. 

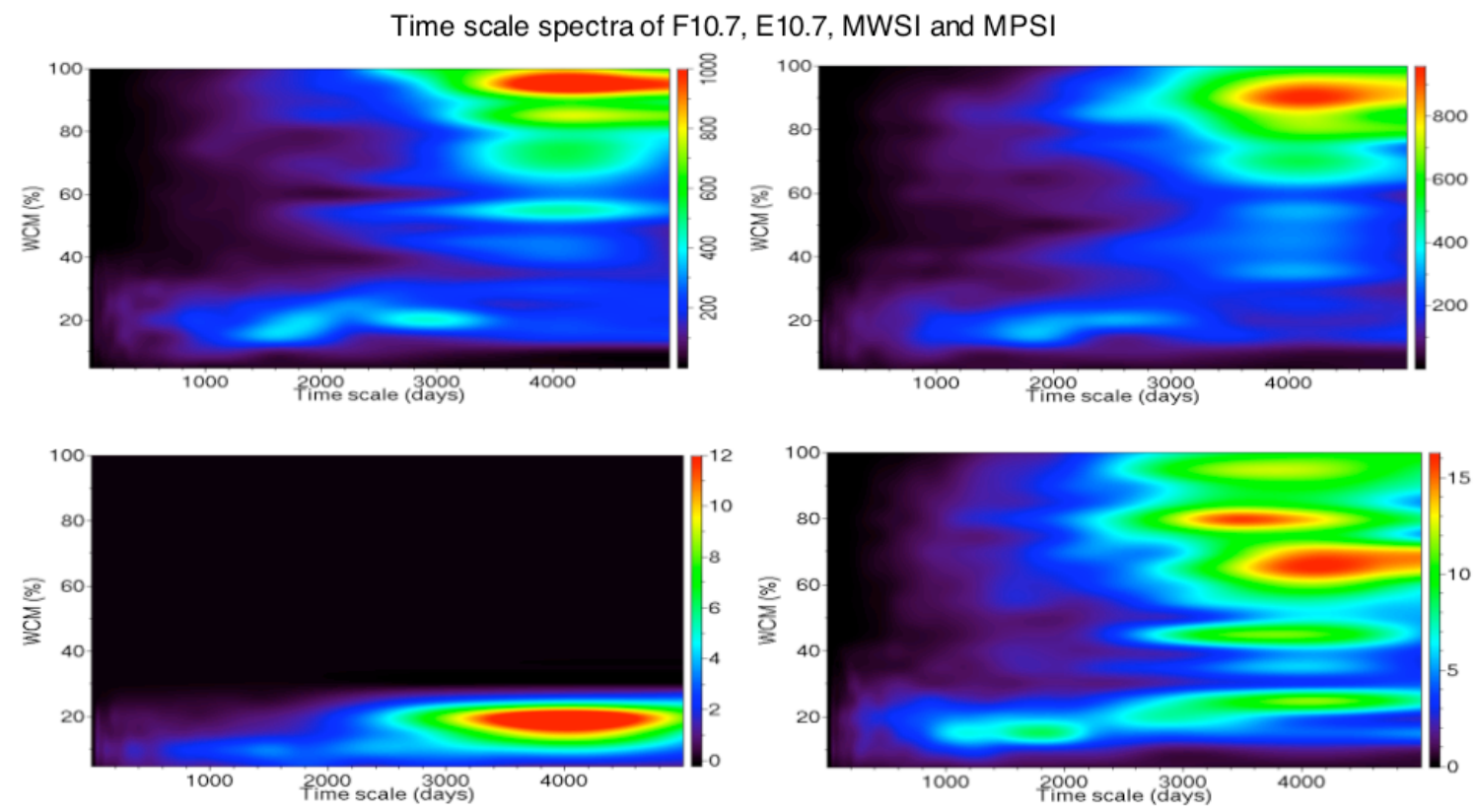

Fig. 6. time-scale spectra, WCM (\%) vs. time scales of F10.7 (upper left), E10.7 (upper right), MWSI (lower left), and MPSI (lower right) for the period 1975 to 2002 .

\section{Discussion and conclusions}

This article presented the first results of a new approach of modelling and forecasting solar activity, as given in Fig. 1. Searching for regularities, periodicities and laws will be done by using neural networks (Fu, 1994). However, it will not be discussed in this article.

New wavelet methods were used to explore indicators of solar activity. By applying time-scale spectra to a set of solar indicators we were able to show both the similarities and differences between these indicators. The time-scale spectra revealed both deterministic and more stochastic features in the variability of the indicators. The 11-year main cycle dominated as expected, but also a more complicated picture appeared. Especially interesting were the time-scale spectra of the Mount Wilson plage index. Further studies clarifying the solar origin will be carried out.

How the time scales varied from 1850 to 2004, for WCMmax, were derived from scalograms of the sunspot number. For WCMmax higher solar activity was, as expected, associated with times of shorter period. However, by comparing the WCM max with the time-scale a more complicated picture appeared (Fig. 3).

Our next step is now to try to explain the results of the wavelet study concerning the background time scales, amplitude and processes. We will construct time series based on solar theory, and then study the series with our wavelet methods.

What can solar dynamo models tell us about the solar activity cycle length, amplitude and processes background? Briefly, a modulation of the 11-year cycle dynamo and cycle length can be achieved by changing the three param- eters; $\omega, \alpha$ and the meridional circulation rate of the dynamo, Dikpati and Gilman (2001); Choudhuri (2003). Dikpati and Charbonneau (1999) emphasized that the velocity of the meridional flow is a critical factor in determining the period of the dynamo cycle. A least-squares fit on their numerical data gave the following scaling law governing the dependence of the dynamo period on model parameters,

$T=56.8 u_{0}^{-0.89} s_{0}^{-0.13} \eta_{T}^{0.22}$,

where the time period $\mathrm{T}$ is measured in years and $u_{0}, s_{0}$ and $\eta_{T}$, i.e. the meridional flow speed, the source coefficient (strength of source term representing the surface generation of poloidal field due to the decay of tilted bipolar active regions) and the turbulent diffusivity, are all measured in cgs units. When they introduced a random variation of the meridional velocity, they found the well-known property that a long cycle follows a short one, but the long-term average was fixed at 11 years by the long-term average velocity of about $17 \mathrm{~m} / \mathrm{s}$. Despite simplified assumptions, for example, that the meridional circulation is just one cell, the results of the models are impressive.

Mundt et al. (1991) found that the solar activity variability, indicated by $R_{z}$, can be described as a low dimension, nonlinear chaotic system of dimension 2.3 , i.e. only three individual variables are needed to describe the variability. Weiss et al. (1984) introduced a complex generalization of three ordinary differential equations first studied by Lorenz (1963) as a model of turbulent convection. Like the Lorenz equations, the equations proposed by Weiss et al. (1984), have chaotic solutions but because they are complex they also have solutions that are periodic in time as the dynamo number is changed. The modulation of the cycle, as, for example, the 
Maunder minimum, is also reproduced. The dynamo number measures the strength of the two induction effects, $\alpha$ and $\Delta \Omega$, relative to the diffusivity. Usoskin et al. (2001), on the other hand, claim that the sunspot activity can be described by three non-chaotic processes; the 22-year solar dynamo field, a weak constant relic field and a randomly fluctuating field. Schrijver and Zwaan (2000) suggest two dynamos: a cycle and a turbulent dynamo. Cognitive models, such as neural network models, will also be used to explain features found in the solar data. Today there are many ways to extract the knowledge that a neural network has learned, Garcez et al. (2002); Fu (1994). In Lundstedt et al. (2002) we first converted the recurrent neural network into a difference equation. We then compared the difference equation with the differential equation describing the solar wind-magnetosphere coupling. Based on the neural network knowledge, we were capable of deriving new knowledge about the decay time of the geomagnetic storm. The recurrent neural network had learned new, interesting physics. Similar comparisons between recurrent neural networks and differential equations describing the solar activity will be carried out. Knowledge and physics can also be coded into the networks (Fu, 1994). The topology is determined by physics laws and knowledge.

The goal of our program (Fig. 1) is to develop a hybrid physics-based neutral network model, explaining and forecasting the solar activity (Lundstedt, 2004). The results from the exploration, using wavelet methods, will be used for neural networks and presented as data and rules. Dynamo theory will be included in the neural networks through comparison with difference equations, as seen in Lundstedt (2001). From helioseismological studies Kosovichev (2003); ChristensenDalsgaard and Thompson (2003); Jensen et al. (2004) information about below the solar surface and on the far side of the Sun will again be presented to the hybrid physics-based neural network.

With this new approach we hope to improve the forecasts of solar activity but also to discover new solar physics.

Acknowledgements. This work is sponsored by the Swedish National Space Board. We are grateful to the following providers of data; ESA/NASA SOHO/MDI team, Wilcox Solar Observatory, Stanford, Mount Wilson Observatory, UCLA, RWC Belgium, Hoyt and Schatten for Rg, NOAA and SEC.

Topical Editor R. Forsyth thanks two referees for their help in evaluating this paper.

\section{References}

Boberg, F., Lundstedt, H., Hoeksema, J. T., Scherrer, P. H., and Lui, W.: Solar mean magnetic field variability: A wavelet approach to WSO and SOHO/MDI observations, J. Geophys. Res., 107(A10), 15-1-15-7, 2002.

Boberg, F. and Lundstedt, H.: Solar wind variation related to fluctuations of the North Atlantic Oscillation, Geophys. Res. Lett., 29(15), 13.1-13.4, 2002.

Choudhuri, A. R.: On the connection between mean field dynamo theory and flux tubes, in press Solar Physics, 215(1), 31-55, 2003.
Christensen-Dalsgaard J. and Thompson, M. J.: Rotation of the solar interior, Dynamic Sun, (Ed.) Dwivedi, B. N., 2003.

Clua de Gonzalez, A. L., Gonzalez, W. D., Dutra, S. L. G., and Tsurutani, B. T.: Periodic Variation in the Geomagnetic Activity: A Study Based on the $A_{p}$ Index, J. Geophys. Res., 98, 92159231, 1993.

Dikpati, M. and Charbonneau, P.: A Babcock-Leighton flux transport dynamo with solar-like differential rotation. Astrophys. J., 518: 508-520, 1999.

Dikpati, M. and Gilman, P.: Flux-transport dynamos with alpha effect from global instability of tachocline differential rotation; a solution for magnetic parity selection in the sun, Astrophys. J., 559, 428-442, 2001.

Duhau, S.: An Early Prediction of Maximum Sunspot Number in Solar Cycle 24, Solar Physics, 213 (1), 203-212, 2003.

Frick, P., Galyagin, D., Hoyt, D. V., Nesme-Ribes, E., Schatten, K. H., Sokoloff, D., and Zakharov, V.: Wavelet analysis of solar activity recorded by sunspot groups, Astron. Astrophys., 328, 670-681, 1997.

Fu, L.-M.: Neural Networks in Computer Intelligence, McGraw-Ill, Inc., 1994.

Garcez, A. S., Broda, K. B., and Gabbay, D. M.: Neural-Symbolic Learning Systems, Foundations and Applications, Springer Verlag, 2002.

Hathaway, D. H., Nandy, D., Wilson, R. M., and Reichmann, E. J.: Evidence that Deep Meridional Flow Sets the Sunspot Cycle Period, Astrophys. J., 589, 665-670. 2003.

Hoyt, D. V. and Schatten, K.: Group Sunspot Numbers: A New Solar Activity Reconstruction, Solar Physics, 181, 491-512, 1998.

Jensen, J. M., Lundstedt, H., Thompson, M. J., Pijpers, F. P., and Rajaguru, S. P.: Applicataion of local-area helioseismic methods as predictors of space weather, in: "Helio- and Asteroseismology: Towards a golden future”, (Ed.) Danesy, D., Proc. SOHO 14/GONG+ 2004 Meeting, ESA SP-559, 497-500, 2004.

Kane, R. P.: Prediction of solar activity: Role of long-term variations, J. Geophys Res., 107, 3-1-3-3, 2002.

Knaack, R. and Stenflo, J. O.: Harmonic Analysis of Solar Magnetic Fields, in: ESA SP-505: SOLMAG 2002, Proceedings of the Magnetic Coupling of the Solar Atmosphere Euroconference, 453-456, 2002.

Kosovichev, A.: Helioseismic tomography, Dynamic Sun, (Ed.) Dwivedi, B. N., Cambridge Univ. Press, 2003.

Krivova, N. A. and Solanki, S. K.: The 1.3 year and 156-day periodicities in sunspot data: Wavelet analysis suggests a common origin, Astron. Astrophys., 394, 701-706, 2002.

Kumar, P. and Foufoula-Georgiou, E.: Wavelet analysis for geophysical applications, Rev. Geophys. 35(4), 385-412, 1997.

Liszka, L.: Cognitive Information Processing in Space Physics and Astrophysics, Pachart Publishing House, Tuscon, 2003.

Lorenz, E. N.: Deterministic nonperiodic flow. J. Atmos. Sci., 20, 130-141, 1963.

Lundstedt, H.: AI Techniques in Geomagnetic Storm Forecasting, in: Magnetic Storm Geophysical Monograph 98, AGU, 1997.

Lundstedt, H.: Solar Activity Predicted with Artificial Intelligence, in Space Weather Geophysical Monograph 125, AGU, 2001.

Lundstedt, H.: Progress in Space Weather Predictions and Applications, in Adv. in Space Res., invited presentation at World Space Conference, COSPAR, Houston, USA, 10-19 October, in press, 2003.

Lundstedt, H.: Solar Activity Modelled and Forecasted: A New Approach, presented at COSPAR meeting in Paris 2004 and submitted to Adv. Space Res., 2004. 
Lundstedt, H., Gleisner, H., and Wintoft, P.: Operational forecasts of geomagnetic $D_{s t}$ index, Geophys. Res. Lett., 29, 34-134-4, 2002.

Mundt, M. D., Maguire II, W. B., and Chase, R. P.: Chaos in sunspot cycle: Analysis and prediction, J. Geophys., Res., 96, 17051716, 1991.

Oliver, R. and Ballester, J. L.: The 158-Day Periodicity in the Rising Phase of Cycle 23, in Proc. SOLSPA: The Second Solar Cycle and Space Weather Euro Conference, Vico Equense, Italy, 24-29 September, 2001, ESA SP-477, February 2002.

Polygiannakis, J., Preka-Papadema, P., and Moussas, X.: On signalnoise decomposition of timeseries using the continuous wavelet transform: application to sunspot index, Mon. Not. R. Astron. Soc., 343, 725-734, 2003.

Rabin, D, DeVore,, C. R, Harvey, K. L., and Hoeksema, J .T.: The Solar Activity Cycle, in The Solar Interior and Atmosphere, (Eds.) Cox, A. N., Livingston, W. C. and Matthews, M. S., Univ. of Arizona Press, 781-843, 1991.

Richardsson, J. D. Paularena, K. I. and Belcher, J. W. A., and Lazarus, A. J.: Solar wind oscillations with a 1.3 year period, Geophys. Res. Lett., 21, 1559-1560, 1994. J. Geophys. Res. Lett., 21, 1559-1560, 1994.

Schatten, K.: Solar activity prediction: Timing predictors and cycle 24, J. Geophys., Res., 107(A11), 15-1-15-7, 2002.

Scherrer, P. H., Wilcox, J. M., Svalgaard, L., Duvall, T. L., Dittmer, P. H., and Gustafson, E. K.: The mean magnetic field of the sun: Observations at Stanford, Solar Physics, 54, 353-361, 1977.

Schrijver C. and Zwaan, C.: Solar and Stellar Magnetic Activity, Cambridge Astrophys. Ser., 34, 172-184, 2000.
Shindell, D. T., Schmidt, G. A., Mann, M. E., Rind, D., and Waple, A.: Solar forcing of regional climate change during the Maunder Minimum, Science 294 , 2149-2152, 2001.

Svalgaard, L., Cliver, E. W, and Kamide, Y.: Sunspot cycle 24: Smallest cycle in 100 years?, Geophys. Res. Lett., 32(L01104), doi:10.1029/2004GL021664, 2005.

Svensmark, H. and Friis-Christensen, E.: Variation of Cosmic Ray Flux and Global Cloud Coverage - A Missing Link in Solar-Climate Relationships, J. of Atmos. and Solar-Terrestrial Physics, 59(11), 1225-1232, 1997.

Tobiska, W. K.: Validating the Solar EUV Proxy, E10.7, J. Geophys. Res., 106(A12), 29 969-29978, 2001.

Torrence, C. and Compo, G. P.: A practical guide to wavelet analysis, Bull. Am. Meteorol. Soc., 79, 61-78, 1998.

Usoskin, I., Mursula, K., and Kovaltsov, G. A.: Simulation of sunspot activity during active Sun and great minima using regular, random and relic fields, Solar Physics, 199, 187-199, 2001.

Usoskin, I. G., Solanki, S. K., Schussler, M., Mursula, K., and Alanko, K.: Millennium-Scale Sunspot Number Reconstruction: Evidence for an Unusually Active Sun since 1940s, Phys. Rev. Lett. 91, 211 101-1-211101-4, 2003.

Weiss, N., Cattaneo, F., and Jones, C. A.: Geophys. Astrophys. Fluid Dyn., 30, 305-341, 1984.

Wernik, A. W., Grzesiak, M., Sadowski, M., and Rothkaehl, H.: Analysis of ionospheric plasma turbulence with the wavelet transform, in: Sadowski, M. and Rothkaehl, H.(Eds.), Proc. Int. Symp. "Plasma 97", Jarnoltowek, 1, 391, Space Res. Center, Polish Academy of Sciences, 1997. 